\title{
Sleep-Disordered Breathing and the Menopausal Transition among Participants in the Sleep in Midlife Women Study
}

\author{
Anna G. Mirer, PhD, MPH ${ }^{1}$, Terry Young, $\mathrm{PhD}^{2}$, Mari Palta, $\mathrm{PhD}^{2}$, Ruth M. Benca, MD, $\mathrm{PhD}^{3}$, \\ Amanda Rasmuson, $\mathbf{M S}^{2}$, and Paul E. Peppard, PhD $^{2}$ \\ ${ }^{1}$ Medical Scientist Training Program, University of Wisconsin School of Medicine and Public \\ Health \\ ${ }^{2}$ Department of Population Health Sciences, University of Wisconsin-Madison \\ ${ }^{3}$ Department of Psychiatry, University of Wisconsin School of Medicine and Public Health
}

\section{Abstract}

Objective-Menopause is widely believed to be an established cause of sleep disorders, but evidence for this theory is inconclusive. Attributing any sleep problem to normal processes of menopause may lead to underdiagnosis of treatable sleep disorders in midlife women. This study uses detailed longitudinal data on sleep and menopausal health from participants in the Sleep in Midlife Women Study to investigate whether risk and severity of sleep-disordered breathing increase with progression through menopause, accounting for changes in age and body habitus.

\begin{abstract}
Methods-219 participants age 38-62 years were recruited from participants in the Wisconsin Sleep Cohort study. Menopausal status was determined from daily diaries in which participants reported menstrual flow, hot flashes, and use of hormonal medications. Each participant underwent in-home polysomnography studies every six months, to measure the apnea-hypopnea index ( $N=1,667$ studies). Linear models with empirical standard errors were fit for logarithm of apneahypopnea index on menopausal status and years in menopause, adjusting for age, BMI, waist girth and neck girth.
\end{abstract}

Results-Compared to participants in premenopause, apnea-hypopnea index was $21 \%$ higher among participants in perimenopause (95\% Confidence Interval $[-4,54]), 31 \%$ higher among participants in postmenopause $([2,68])$, and $41 \%$ higher among participants whose menopausal stage could not be distinguished between peri- and postmenopause $([8,82])$. Among women who had begun perimenopause, each additional year in menopause was associated with $4 \%$ greater AHI $([2,6])$.

Conclusions-Progression through menopause is associated with greater SDB severity. This association is independent of aging and changes in body habitus.

Corresponding Author: Paul E. Peppard, 611A WARF Building, 610 Walnut St. Madison, WI 53726, Phone: (608) 262-2680, Fax: (608) 262-4226, ppeppard@wisc.edu.

Conflicts of Interest: None

Disclaimers: None 


\section{Keywords}

Menopause; Sleep Apnea Syndromes; Sleep Apnea; Obstructive; Women's Health; Aging

\section{Introduction}

Among younger adults, the prevalence of sleep apnea in men is roughly three times the prevalence in women, but the effect of gender is smaller among older adults. ${ }^{1-4}$ A possible explanation for the contrast in aging patterns between genders is that the transition to menopause may increase risk of sleep-disordered breathing. Several studies have attempted to investigate the association between menopausal status and sleep-disordered breathing, but those studies had important methodological limitations.

Studies designed to accurately measure menopause are rarely designed to fully measure sleep-disordered breathing, and vice versa, making prior results difficult to interpret. Classification of menopausal status relies on menstrual history, which is difficult to recall retrospectively. Since sleep-disordered breathing manifests during sleep-when patients are rarely observed, and cannot observe themselves at all-underdiagnosis is common. Selfreport of sleep-disordered breathing is therefore of limited value, and likely to introduce bias as some groups of people are more likely than others to be accurately diagnosed.

Many studies have failed to account for confounding, especially by age. Menopause is an aging process, and since age is a powerful predictor of sleep apnea risk and severity, it is important to distinguish whether menopause itself is associated with greater risk of sleep apnea or whether it simply captures an aging process similar to that in men. Some studies found that more advanced menopausal stage is associated with sleep-disordered breathing, but failed to account for the effect of aging. ${ }^{5-10}$

A further consideration is that if menopausal status does affect breathing during sleep, it might be expected to do so primarily through changes in body habitus. Postmenopausal women tend to have higher BMI and central adiposity, ${ }^{11}$ both of which are strongly associated with SDB. ${ }^{12}$ If any association between menopausal stage and sleep-disordered breathing can be explained either by body habitus changes or by aging itself, then there is little immediate clinical utility in using menopausal status as a risk factor-knowing a patient's age and BMI would suffice to assess her risk.

More rigorous studies accounting for both age and body habitus have yielded conflicting results, with some showing an association independent of age, ${ }^{13-16}$ and others showing no association. ${ }^{17}$ Among population-based epidemiologic studies, only one prior investigation ${ }^{13}$ has followed the same women through different menopausal stages. This investigation by Young et. al. using data from the Wisconsin Sleep Cohort found a smaller estimated association between menopause and SDB severity (compared to participants in premenopause, odds ratios of 1.66 for perimenopause, 3.22 for postmenopause, and 2.82 for undetermined peri-postmenopause) than was suggested by studies that used cross-sectional data. ${ }^{14,16}$ This discrepancy may be a result of study design, as the longitudinal studies were able to control for time-invariant factors intrinsic to each participant whereas the cross- 
sectional studies are limited to comparing difference across different women. However the Young et. al. study was limited by the small number of observations on each participant, and an average five-year gap between observations.

The present study investigates whether progression through menopause is associated with risk and severity of sleep-disordered breathing, independent of age and changes in body habitus, in order to address the question of whether menopause itself is a useful marker of SDB risk and severity. It uses data from the Sleep in Midlife Women Study, a populationbased cohort study designed to gather high-quality, precise longitudinal data on both menopausal status and sleep-disordered breathing.

\section{Methods}

\section{Study Design}

Sleep in Midlife Women Study participants were recruited from among female participants in the Wisconsin Sleep Cohort Study. The parent study's design is described in full elsewhere. ${ }^{3}$ Briefly, Wisconsin state workers were sampled at random and recruited to participate in a mailed survey from 1989-1993. Among survey responders, a stratified random sample was selected for further study. Sampling weights were designed to enrich the sample with participants with sleep apnea. From 1989-2003, this subsample of responders was invited to undergo in-laboratory polysomnography, and to return for sleep studies approximately every four years, continuing through the present. From 1996-2005, all female participants who were "at risk" for menopause (defined as having begun Perimenopause or being over 47 years old) were invited to participate in the Sleep in Midlife Women Study. Because the median age in the cohort was 47 at the start of recruitment, women who had already begun perimenopause were prioritized, in order to maximize the number of observations on women not yet in postmenopause. The response rate was approximately $80 \%$.

Sleep in Midlife Women Study participants completed diaries, including daily data on sleep symptoms and menstrual symptoms, and monthly data on hormonal medication use. Participants also underwent polysomnography studies in their own homes approximately every six months through 2007. On the night of the sleep study, an interview was conducted including questions on alcohol use and smoking, and weight, waist girth, and neck girth measurements were taken. BMI was calculated using height measured at the participant's most recent laboratory visit.

Protocols and informed consent documents for the Sleep in Midlife Women Study and Wisconsin Sleep Cohort Study were approved by the University of Wisconsin-Madison Health Sciences Institutional Review Board.

\section{Sleep-Disordered Breathing Measures}

Sleep-disordered breathing was assessed by measuring the apnea-hypopnea index (AHI) to indicate the rate of breathing pauses during sleep. In-home polysomnography (P-series, Compumedics USA, Inc., Fridley, MN) included piezoelectric chest and abdominal bands to record breathing effort, nasal-oral thermistry to detect airflow, and finger-pulse oximetry to 
record arterial oxygen saturation. Apnea-hypopnea index was calculated by summing the number of apneas (air flow cessation $\geq 10$ seconds) and hypopneas (25\% decrease in airflow, or interruption in flow pattern, for $\geq 10$ seconds, with oxygen desaturation of $\geq 4 \%$ ), divided by objectively measured total sleep time.

\section{Menopausal stage measures}

Menopausal stage was classified using criteria consistent with the Stages of Reproductive Aging Workshop. ${ }^{18}$ Menstrual history was used wherever possible to date age at early perimenopause, age at late perimenopause and age and postmenopause. History of hysterectomy and/or oophorectomy were also used where relevant. Where menstrual history was missing or uninformative, criteria based on use of hormonal medications or chronological age were used. Each participant was classified as being premenopausal until at least one criterion for being in perimenopause was met, then classified as perimenopausal until one of the criteria for postmenopause was met. A full list of criteria is presented in Table 1. Initially perimenopause was divided into early perimenopause and late perimenopause. However AHI was very similar in the two perimenopause categories, absolutely and conditional on the covariates of interest for this analysis, so the two categories were combined post hoc into a single perimenopause category. Following the method of Young et. al., ${ }^{13}$ a fourth category of menopausal status was created for observations that were known to have occurred after the start of perimenopause, but at which perimenopause could not be distinguished from postmenopause. Continuous time in menopause was estimated as time elapsed since the date of the start of early perimenopause.

\section{Statistical Analysis}

Descriptive statistics were calculated as means and standard deviations of continuous variables, and frequencies of categorical variables. All analyses were conducted using SAS version 9.3 (Cary, NC).

The relationships of menopausal stage and time in menopause to AHI were modeled using linear and logistic regressions. Initially logistic models using cut points of AHI greater than 10, 15, and 30 were compared, and results were found to be similar. Models using AHI $\geq 15$ are presented here as that cutoff is commonly used in clinical diagnosis of OSA. The distribution of AHI was right skewed in this healthy population, so for linear models we transformed AHI by taking the natural logarithm, adding one to preserve zero values. Thus the outcome in linear models was $\ln (\mathrm{AHI}+1)$. Linear regression coefficients were then exponentiated to produce a ratio of AHI +1 . Results of these models are presented as the ratios, which may also be used to obtain percent differences. Models using continuous time in menopause as a predictor excluded observations on participants in premenopause and observations on participants whose start of early perimenopause could not be dated, either because last menstrual period predated the study, because prospective data was missing, or because subjects did not meet criteria outlined in Table 1 .

Mixed effects models with random intercepts, using empirical standard errors to account for repeated measures, were run using the MIXED procedure. Each model was adjusted for age, BMI, waist girth, neck girth, hormone therapy use, alcohol use, and smoking history. 
Observations with missing covariate data were excluded. An overall $F$-test and a test for linear trend were calculated for menopausal stage.

Effect modification was assessed by interacting menopausal status and time in menopause with several different covariates: age, years in postmenopause, use of menopausal hormone therapy, BMI, waist circumference, and neck circumference. For each assessment, product terms were included in fully adjusted linear models, and $P$-values were assessed on individual product terms and on the overall $F$-test for all product terms in the model. $P$ values below 0.01 were considered evidence consistent with effect modification.

Logistic regression models with outcomes of AHI $\geq 15$ were adjusted for the same covariates. Generalized Estimating Equations, assuming compound symmetry, were used to account for repeated measures, via the GENMOD procedure.

\section{Results}

\section{Sample characteristics}

Of the 239 participants recruited for the Sleep in Midlife Women Study, 219 participants had nonmissing values of all covariates for at least one visit. 1,667 sleep studies were included in this analysis. Participant characteristics at baseline are presented in Table 2.

Mean AHI was relatively high, reflecting the fact that the in-home polysomnography equipment measured AHI systematically higher than typical laboratory equipment. There were relatively few observations on participants in premenopause, as a result of the study design that prioritized recruitment of women who had begun perimenopause. Women who had progressed further through menopause by the beginning of the study were older and had higher mean AHI. Women beginning the study in premenopause had smaller mean waist circumference, reported more alcohol consumption, and had less history of smoking.

\section{Regression results}

Full results of multivariable regressions of AHI on menopausal stage are presented in Table 3. There was a monotonic increase in AHI from premenopause, to perimenopause, and postmenopause. However, the highest AHI was observed in the undetermined peripostmenopause category. This relationship was independent of chronologic age and body habitus. Though age, BMI, neck girth, and waist girth were also associated with higher AHI, these factors did not explain the association with menopausal stage. A similar pattern across categories of menopausal stage was found in the logistic model, but confidence intervals were wide.

Results of modeling regression of AHI on years in menopause are shown in Table 4. Every additional year in menopause was associated with $4 \%$ higher AHI (95\% confidence interval [2\%-6\%]). This association was also independent of age and body habitus measures. Each additional year in menopause was associated with $7 \%$ greater odds of having AHI of fifteen or greater, but with a confidence interval that includes one. This suggests an exposureresponse relationship, that is that the longer participants had been in menopause, the more severe was their SDB on average. 
There was no evidence that menopausal stage was modified by age, by any measure of body habitus, or by menopausal hormone therapy use (see Supplemental Digital Content 1). There was also no evidence that the association between menopausal stage and AHI was modified by time in menopause.

\section{Discussion}

Our analysis found that the transition from premenopause to postmenopause is associated with increased severity of sleep-disordered breathing, and that these changes are not entirely explained by chronological aging, or by changes in body habitus. We also found evidence of an exposure-response relationship between progression through menopause and sleepdisordered breathing, in which mean $\mathrm{AHI}$ in perimenopause was in between mean $\mathrm{AHI}$ in premenopause or postmenopause. The estimated comparison between premenopause and perimenopause was not sufficiently precise to discount association due to chance, in that the confidence interval included one. However the association between continuous time in menopause and higher AHI also supports an exposure-response model.

Though BMI, waist circumference, and neck circumference were all associated with higher AHI, we did not find evidence that changes in body habitus are the sole mechanism through which menopausal stage could affect AHI. Many other mechanisms by which menopause could affect sleep-disordered breathing are possible, but they have not been rigorously tested. Levels of sex hormones may be implicated, since ovarian aging is associated with lower levels of estrogens and progesterones. Some short-term experiments on small samples have suggested that level of sex hormone can affect breathing during sleep. ${ }^{21,22}$ However exogenous hormones have not been conclusively shown to be beneficial to risk or severity of sleep apnea, ${ }^{23}$ suggesting that some other aspect of the menopausal transition may be at work.

These findings agree with much of the published literature in finding a positive association between later menopausal status and SDB. However they give stronger support to a causal association. In contrast to the studies by Bixler et. $\mathrm{al}^{14}$ and Polesel et. al. ${ }^{16}$, this study used repeated measures that allow regression modeling to control for time-invariant personal characteristics that could bias a cross-sectional association. One notable different between these studies and ours is that the magnitude of the associations found in this analysis were substantially smaller.

The finding that menopausal hormone therapy did not modify the association between menopausal stage and AHI contradicts findings from an earlier study of data from the Wisconsin Sleep Cohort Study. ${ }^{13}$ The discrepancy may be explained by the fact that data analyzed for the cited paper by Young et. al. was collected prior to 2002, a year when the results of the Women's Health Initiative study led to an abrupt shift in prescribing practices away from long-term chronic-disease prevention and toward short-term symptomatic treatment. $^{20}$

It is surprising that the most severe sleep-disordered breathing was observed in the group with undetermined menopausal status. If this group represents a mix of participants whose 
"true" menopausal status is perimenopause and participants in "true" postmenopause, we would expect the estimated association with AHI to fall in between that of perimenopause and postmenopause. It is unclear why this group should be at especially high risk of sleep apnea. One possibility is that women who had irregular menses or oligomenorrhea throughout adulthood may have been difficult to classify on the basis of their menstrual histories, or may have had particularly long perimenopauses that lasted beyond the end of the study, leading to their data being systematically more likely to be missing. This could represent a qualitatively different experience of menopause, with some biological importance. Our study did not collect data on polycystic ovary syndrome (PCOS), and this is one known predictor of irregular menses. Several studies of women with PCOS have found that they are at greater risk of obstructive sleep apnea, independent of age and BMI. ${ }^{19}$ Another possible explanation is simply "noisy" data-the confidence intervals around the odds ratio estimate for the undetermined group do not preclude an elevated "risk" intermediate to that of the perimenopause and postmenopause groups.

\section{Strengths and Limitations}

This project's findings present the most comprehensive study of menopause and SDB to date. This study has several important strengths that distinguish it from existing studies. Very few studies have been designed to both measure menopause well and measure SDB well. Our study measured menopause with high precision, with participants reporting menstrual and symptom data daily. Furthermore the measurements used to define menopausal status were collected prospectively, rather than relying on recall, which can be inaccurate. A third strength is the length of follow-up time, which allowed many participants to be observed at different stages of the menopausal transition, rather than relying solely on comparisons across different women. This study is also notable for its ability to control for factors associated with menopause, including age, body habitus measures, and health behaviors such as alcohol and smoking.

It was necessary in this study to prioritize women already in perimenopause for recruitment in order to avoid losing the chance to observe them before their transition to menopause was complete, but the small number of observations on participants in premenopause is an important limitation. It is possible that small sample size contributed to a lack of precision on the estimated difference in mean AHI compared to women in perimenopause. It is possible that greater sample size would have produced a clearer contrast. Small sample size also likely contributed to the broad confidence intervals in the logistic models.

Only seven of the participants who contributed observations to this analysis were women of color. Thus, we did not have the ability to examine whether race and ethnicity modified the association of menopausal stage with sleep-disordered breathing, and the generalizability of our finding to women of color is limited.

Lastly, while our method of staging menopause was able to describe a process that was associated with SDB, the boundaries between menopausal categories are always somewhat arbitrary. This limitation could be conceptualized as a type of measurement error, but that assumes that each participant has a "true" menopausal status, which could in theory be measured with precision. The heterogeneity of the menopausal experience, however, 
challenges that assumption. Any attempt to quantify and categorize menopause as a single, universal process is inherently limited.

\section{Conclusions}

In this population of mid-life women, menopause was a risk factor for sleep-disordered breathing, independent of age and body habitus. Later menopausal stage and time in menopause were both associated with higher AHI, suggesting an exposure-response relationship between further progression through menopause and sleep-disordered breathing severity.

\section{Supplementary Material}

Refer to Web version on PubMed Central for supplementary material.

\section{Acknowledgments}

We thank Laurel A. Finn and Jodi H. Barnet for their help preparing this article. We thank Diane Austin, Diane Demonoco Dowd, Mari Dresner, Crystal Halvorson, Kathryn Hoffman, Angie Kujak, Jennifer Kujak, Andrea Peterson, Angela Slattery, and Rebecca Swain-Eng for their work on the Sleep in Midlife Women Study.

Financial Support: This work was supported by the National Institute of Aging (R01AG14124), National Heart, Lung, and Blood Institute (R01HL62252) and the National Center for Research Resources (1UL1RR025011) at the National Institutes of Health. Anna G. Mirer is in the University of Wisconsin Medical Scientist Training Program (T32GM008692)

\section{References}

1. Redline S, Kump K, Tishler PV, Browner I, Ferrette V. Gender differences in sleep disordered breathing in a community-based sample. Am J Respir Crit Care Med. 1994; 149(3 Pt 1):722-726. [PubMed: 8118642]

2. Young T, Skatrud J, Peppard PE. Risk factors for obstructive sleep apnea in adults. JAMA. 2004; 291(16):2013-2016. [PubMed: 15113821]

3. Peppard PE, Young T, Barnet JH, Palta M, Hagen EW, Hla KM. Increased Prevalence of SleepDisordered Breathing in Adults. Am J Epidemiol. 2013; 177(9):1006-1014. [PubMed: 23589584]

4. Young T, Palta M, Dempsey J, Skatrud J, Weber S, Badr S. The occurrence of sleep-disordered breathing among middle-aged adults. N Engl J Med. 1993; 328(17):1230-1235. [PubMed: 8464434]

5. Tantrakul V, Guilleminault C. Chronic sleep complaints in premenopausal women and their association with sleep-disordered breathing. Lung. 2009; 187(2):82-92. [PubMed: 19219502]

6. Hachul H, Bittencourt LRA, Soares JM Jr, Tufik S, Baracat EC. Sleep in post-menopausal women: Differences between early and late post-menopause. Eur J Obstet Gynecol Reprod Biol. 2009; 145(1):81-84. [PubMed: 19414210]

7. Resta O, Caratozzolo G, Pannacciulli N, et al. Gender, age and menopause effects on the prevalence and the characteristics of obstructive sleep apnea in obesity. Eur J Clin Invest. 2003; 33(12):10841089. [PubMed: 14636291]

8. Dancey DR, Hanly PJ, Soong C, Lee B, Hoffstein V. Impact of menopause on the prevalence and severity of sleep apnea. Chest. 2001; 120(1):151-155. [PubMed: 11451831]

9. Resta O, Bonfitto P, Sabato R, De Pergola G, Barbaro MPF. Prevalence of obstructive sleep apnoea in a sample of obese women: effect of menopause. Diabetes Nutr Metab. 2004; 17(5):296-303. [PubMed: 16295052] 
10. Anttalainen U, Saaresranta T, Aittokallio J, et al. Impact of menopause on the manifestation and severity of sleep-disordered breathing. Acta Obstet Gynecol Scand. 2006; 85(11):1381-1388. [PubMed: 17091421]

11. Davis SR, Castelo-Branco C, Chedraui $\mathrm{P}$, et al. Understanding weight gain at menopause. Climacteric. 2012; 15(5):419-429. [PubMed: 22978257]

12. Young T, Shahar E, Nieto FJ, et al. Predictors of sleep-disordered breathing in communitydwelling adults: the Sleep Heart Health Study. Arch Intern Med. 2002; 162(8):893-900. [PubMed: 11966340]

13. Young T, Finn L, Austin D, Peterson A. Menopausal status and sleep-disordered breathing in the Wisconsin Sleep Cohort Study. Am J Respir Crit Care Med. 2003; 167(9):1181-1185. [PubMed: 12615621]

14. Bixler EO, Vgontzas AN, Lin HM, et al. Prevalence of sleep-disordered breathing in women: effects of gender. Am J Respir Crit Care Med. 2001; 163(3 Pt 1):608-613. [PubMed: 11254512]

15. Rowley JA, Zhou XS, Diamond MP, Badr MS. The determinants of the apnea threshold during NREM sleep in normal subjects. Sleep. 2006; 29(1):95-103. [PubMed: 16453986]

16. Polesel DN, Hirotsu C, Nozoe KT, et al. Waist circumference and postmenopause stages as the main associated factors for sleep apnea in women: a cross-sectional population-based study. Menopause. Feb.2015

17. Carskadon MA, Bearpark HM, Sharkey KM, et al. Effects of menopause and nasal occlusion on breathing during sleep. Am J Respir Crit Care Med. 1997; 155(1):205-210. [PubMed: 9001313]

18. Harlow SD, Gass M, Hall JE, et al. Executive summary of the Stages of Reproductive Aging Workshop +10: addressing the unfinished agenda of staging reproductive aging. Climacteric. 2012; 15(2):105-114. [PubMed: 22338612]

19. Ehrmann DA. Metabolic dysfunction in PCOS: Relationship to obstructive sleep apnea. Steroids. 2012; 77(4):290-294. [PubMed: 22178788]

20. Hersh AL, Stefanick ML, Stafford RS. National use of postmenopausal hormone therapy: annual trends and response to recent evidence. JAMA. 2004; 291(1):47-53. [PubMed: 14709575]

21. Netzer NC, Eliasson AH, Strohl KP. Women with sleep apnea have lower levels of sex hormones. Sleep Breath. 2003; 7(1):25-29. [PubMed: 12712394]

22. D'Ambrosio C, Stachenfeld NS, Pisani M, Mohsenin V. Sleep, breathing, and menopause: the effect of fluctuating estrogen and progesterone on sleep and breathing in women. Gend Med. 2005; 2(4):238-245. [PubMed: 16464735]

23. Mirer AG, Peppard PE, Palta M, Benca RM, Rasmuson A, Young T. Menopausal hormone therapy and sleep-disordered breathing: evidence for a healthy user bias. Ann Epidemiol. 2015; 25(10): 779-784.e1. [PubMed: 26358364] 


\section{Table 1}

\section{Classification of Menopausal Stage}

\begin{tabular}{l|l}
\hline Start of Perimenopause & Start of Postmenopause \\
\hline $\begin{array}{l}\text { First incidence of no flow with no hormonal contraception } \\
\text { Change in cycle length } \geq 7 \text { days with no hormonal contraception }\end{array}$ & $\begin{array}{l}\text { Beginning of } 12 \text { months of no flow } \\
\text { Beginning of } 6 \text { months of no flow while using menopausal hormone } \\
\text { therapy } \\
\text { Menopausal hormone therapy for } \geq 12 \text { months }\end{array}$ \\
$\begin{array}{l}\text { First incidence of hot flashes or night sweats while using hormonal } \\
\text { Contraception } \\
\text { incidence of hot flashes or night sweats }\end{array}$ & $\begin{array}{l}60^{\text {th }} \text { Birthday } \\
55^{\text {th }} \text { Birthday with menopausal hormone therapy } \\
\text { FSH }>40 \text { after ovary-sparing surgery } \\
\text { Ovary-removing surgery } 6 \text { or more months ago }\end{array}$ \\
\hline
\end{tabular}




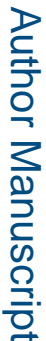

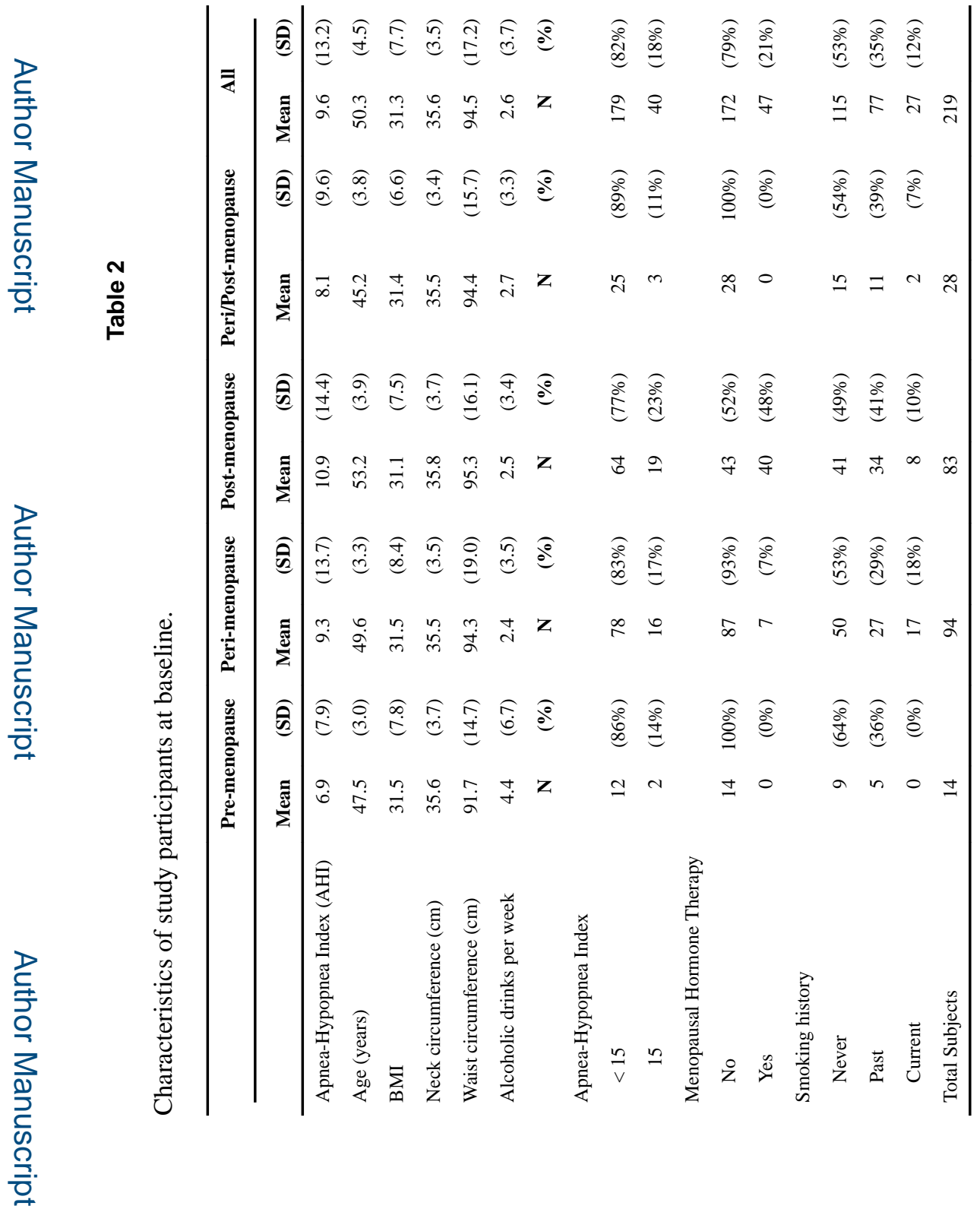

Menopause. Author manuscript; available in PMC 2018 February 01. 


\section{Table 3}

Results of multivariable linear (outcome $=\log (\mathrm{AHI}+1)$ ) and logistic (outcome $=\mathrm{AHI} 215)$ regression of AHI on menopausal stage, with and without adjustment.

\begin{tabular}{|c|c|c|c|c|}
\hline & \multicolumn{2}{|c|}{$\begin{array}{l}\text { Linear } \\
\text { AHI Ratio (95\% CI) }\end{array}$} & \multicolumn{2}{|c|}{$\begin{array}{l}\text { Logistic } \\
\text { Odds Ratio (95\% CI) }\end{array}$} \\
\hline & $\mathrm{N}=1,886$ & $\mathrm{~N}=1,667$ & & $N=1,667$ \\
\hline \multicolumn{5}{|l|}{ Menopausal stage } \\
\hline Premenopause & 1.00 (ref.) & 1.00 (ref.) & 1.00 (ref.) & 1.00 (ref.) \\
\hline Perimenopause & $1.21(1.00,1.46)$ & $1.21(0.96,1.54)$ & $1.22(0.61,2.46)$ & $1.13(0.31,4.13)$ \\
\hline Postmenopause & $1.61(1.31,1.97)$ & $1.31(1.02,1.68)$ & $1.75(0.83,3.70)$ & $1.31(0.33,5.15)$ \\
\hline Peri- to Postmenopause (undetermined) & $1.48(1.20,1.82)$ & $1.41(1.08,1.82)$ & $1.58(0.74,3.37)$ & $1.52(0.39,5.87)$ \\
\hline Overall $F$ test & $P<0.01$ & $P=\mathbf{0 . 0 3}$ & $P<0.01$ & $P=0.63$ \\
\hline Age (years) & -- & $1.05(1.03,1.07)$ & -- & $1.09(1.03,1.16)$ \\
\hline BMI & -- & $1.03(1.00,1.05)$ & -- & $1.07(1.00,1.14)$ \\
\hline Neck circumference $(\mathrm{cm})$ & -- & $1.04(1.02,1.06)$ & -- & $1.09(1.00,1.19)$ \\
\hline Waist circumference $(\mathrm{cm})$ & -- & $1.01(1.01,1.02)$ & -- & $1.04(1.01,1.07)$ \\
\hline Menopausal Hormone Therapy & -- & & -- & \\
\hline No & -- & 1.00 (ref.) & -- & 1.00 (ref.) \\
\hline Yes & -- & $0.96(0.86,1.08)$ & -- & $0.83(0.46,1.49)$ \\
\hline Alcoholic drinks per week & -- & $1.00(0.99,1.02)$ & -- & $1.04(0.98,1.10)$ \\
\hline Smoking history & -- & & -- & \\
\hline Never & -- & 1.00 (ref.) & -- & 1.00 (ref.) \\
\hline Past & -- & $0.90(0.77,1.04)$ & -- & $0.61(0.34,1.09)$ \\
\hline Current & -- & $0.95(0.80,1.13)$ & -- & $0.69(0.33,1.44)$ \\
\hline
\end{tabular}




\section{Table 4}

Results of multivariable linear (outcome $=\log (\mathrm{AHI}+1)$ ) and logistic (outcome $=\mathrm{AHI} \geq 15$ ) regressions of AHI on continuous time in menopause. Observations on subjects in premenopause or whose date of beginning perimenopause was unknown were excluded.

\begin{tabular}{lll}
\hline & Linear & Logistic \\
& AHI Ratio $(\mathbf{9 5 \%}$ CI $)$ & Odds Ratio (95\% CI) \\
& N=1,391 & N=1,391 \\
\hline Years since start of perimenopause & $\mathbf{1 . 0 4}(\mathbf{1 . 0 2}, \mathbf{1 . 0 6})$ & $\mathbf{1 . 0 7}(\mathbf{0 . 9 7 , 1 . 1 8})$ \\
Age (years) & $1.03(1.01,1.05)$ & $1.07(0.99,1.16)$ \\
BMI & $1.02(0.99,1.05)$ & $1.04(0.97,1.11)$ \\
Waist circumference (cm) & $1.02(1.01,1.03)$ & $1.10(1.01,1.21)$ \\
Neck circumference $(\mathrm{cm})$ & $1.03(1.01,1.05)$ & $1.05(1.02,1.08)$ \\
Menopausal Hormone Therapy & & \\
No & $1.00($ ref. $)$ & $1.00($ ref. $)$ \\
Yes & $0.98(0.88,1.09)$ & $0.74(0.38,1.41)$ \\
Alcoholic drinks per week & $1.00(0.99,1.01)$ & $1.02(0.95,1.09)$ \\
Smoking history & & $1.00($ ref. $)$ \\
Never smoker & $1.00($ ref. $)$ & $0.51(0.26,0.98)$ \\
Past smoker & $0.88(0.74,1.03)$ & $0.68(0.31,1.47)$ \\
Current smoker & $0.94(0.78,1.13)$ & \\
\hline
\end{tabular}

